\title{
Factores pronósticos en cáncer renal localizado y localmente avanzado
}

\author{
Rodríguez Jasso VH, Serrano Brambila E, Maldonado Alcaraz E. \\ Servicio de Urología. Hospital de Oncología Centro Médico Nacional Siglo XXI. \\ Instituto Mexicano del Seguro Social. México. \\ Actas Urol Esp. 2008;32(3):320-324
}

\section{RESUMEN}

FACTORES PRONÓSTICOS EN CÁNCER RENAL LOCALIZADO Y LOCALMENTE AVANZADO

En los últimos 10 años se han identificado diversos factores que confieren efecto pronóstico en el cáncer renal. El estadio patológico, el grado nuclear e histológico, son los más frecuentemente estudiados y los más importantes en este momento. Evaluamos esos factores y agregamos otras variables, en un intento por encontrar nuevos parámetros que pudieran ser de utilidad. Se incluyeron para el presente estudio 96 casos de cáncer de células renales no metastásico. Encontramos como se menciona por otros autores que el estadio patológico y de Furhman son los factores pronósticos más fuertes, pero la presencia de tumor palpable, dolor o pérdida de peso también tuvieron significancia estadística.

Palabras clave: Cáncer renal. Factores pronósticos.

\section{ABSTRACT}

PROGNOSTIC FACTORS IN LOCALISED AND REGIONALLY ADVANCED RENAL CELL CARCINOMA In the last 10 years, several factors have been identified to confer a prognostic effect on renal cancer outcome. Pathologic stage, nuclear and histologic grade are the most frecuent studied and the most important at this moment. We evaluated those factors and introduced some others, looking for new parameters that could be useful. 96 cases of non methastatic renal cell cancer were included in our study. We found that as was mentioned by other authors pathologic and Furhman stage are the stronger prognostic factors but the presence of palpable tumor, pain and weight lost had significance too.

Keywords: Renal cancer. Prognostic factors.

$\mathrm{S}$

e estima que existen 80.000 a 100.000 casos nuevos de cáncer renal diagnosticados en el mundo cada año, representando aproximadamente $3 \%$ de todas las neoplasias en adultos. Y se considera el cáncer más letal en urología con 12.000 muertes al año en $\mathrm{EU}^{1}$.

La relación hombre-mujer es de 2:1 y ocurre entre la quinta y séptima década de la vida ${ }^{2}$. El antecedente de tabaquismo está presente en 30\% de los tumores renales ${ }^{3}$.

Aproximadamente el 40 a $50 \%$ de los pacientes con cáncer renal están asintomáticos al momento de la presentación y son diagnosticados incidentalmente por TAC o USG llevados a cabo por sintomas no relacionados ${ }^{1}$.
La hematuria ocurre en el 40-60\% de los pacientes, dolor en flanco en 35-40\% y masa palpable esta presente en $25-50 \%$ de los casos. Esta triada clásica ocurre en menos del $10 \%$ de los $\operatorname{casos}^{4,5}$.

La hipercalcemia como síndrome paraneoplásico ocurre en el 13,9\% y la elevación del calcio sérico es secundaria a la producción de péptidos proteicos similares a la hormona paratiroidea por el tumor, y la actividad osteolítica por las metástasis óseas ${ }^{4,7,9,12}$

Un tercio de los pacientes diagnosticados con cáncer renal tendrán enfermedad metastásica al momento del diagnóstico y más de la mitad de los pacientes tratados por enfermedad localizada eventualmente tendrán recaída ${ }^{6,13}$. 
Como en cualquier otra neoplasia se deben determinar las características del tumor, las características propias del paciente y correlacionar estos con el pronóstico, esto no sólo es útil para el tratamiento sino para estimar la evolución ${ }^{7-9,15,19}$

Durante los últimos 10 años muchas variables han sido probadas en un intento de identificar aquellas con mayor correlación pronóstica para carcinoma renal ${ }^{9}$

Los factores pronósticos importantes incluyen signos y síntomas específicos, aquellos relacionados con el tumor y exámenes de laboratorio. Los factores relacionados con el tumor como: Estadio patológico, Tamaño, Grado nuclear y Subtipo histológico han demostrado ser los más útiles ${ }^{10,20}$

El estadio tumoral, que refleja la diseminación anatómica y el desarrollo de la enfermedad, es reconocido como el factor pronóstico más importante para determinar la conducta clínica y el resultado de carcinoma renal ${ }^{9,14,16,20}$

Se han demostrado tasas de sobrevida a 5 años de 70 a 90\% para tumores organoconfinados y reducción de 15 a $20 \%$ en la sobrevida cuando existe invasión a la grasa perinefrítica ${ }^{10,16}$

La tasa de sobrevida a 5 años en pacientes con trombo en vena es de $45-60 \%$ siempre y cuando el tumor se encuentre confinado al riñón ${ }^{3}$.

En relación al tamaño del tumor se ha observado una importante disminución en la sobrevida en aquellos pacientes con tumores grandes; tumores menores de $7 \mathrm{~cm}$ tienen una sobrevida a 5 años de $84 \%$, mientras que tumores de 7-10 cm tienen una sobrevida de $50 \%$ y tumores de más de $10 \mathrm{~cm} \mathrm{0 \%} \mathrm{a} 5$ años ${ }^{4,8}$

La importancia del grado nuclear como factor pronóstico se observa en el trabajo original de Fuhrman, quien reportó tasas de sobrevida a 5 años de $64 \%$ para grado $1,34 \%$ para grado $2,31 \%$ para grado 3 , y $10 \%$ para grado $4^{7}$.

El tipo histológico más frecuente es el carcinoma de células claras (70-80\%), también es el que tiene un comportamiento más benigno; el carcinoma papilar se presenta en 10-15\% de los casos. El carcinoma de células cromófobas representa el 5\% y aunado con el carcinoma de conductos colectores (1\%) son las estirpes histológicas más agresivas, además de la diferenciación sarcomatoide ${ }^{15,18}$.

El identificar cuales son los factores pronósticos y su papel en el desenlace de los pacientes con cáncer renal de nuestra es de primordial importancia por las razones antes citadas.

\section{MATERIAL Y MÉTODOS}

Con el objetivo de evaluar el impacto clínico de los factores pronósticos en pacientes con cáncer renal localizado y localmente avanzado realizamos una análisis retrospectivo de expedientes clínicos de todos los pacientes con éste diagnóstico en el servicio de Urología del HECMNSXXI en un periodo comprendido de enero de 1998 a diciembre del 2002.

Se buscó intencionadamente en cada uno de los expedientes la edad, tabaquismo, estadio patológico pre y postoperatorio, tipo histológico, grado histológico, y presencia al diagnóstico de hematuria, masa palpable, dolor, hipercalcemia o pérdida de peso.

Se excluyeron aquellos pacientes con enfermedad metastásica al momento del diagnóstico, los que recibieron tratamiento neoadyuvante o adyuvante y los que abandonaron su seguimiento o no tuvieron expediente localizable.

\section{Análisis estadístico}

Se analizaron tablas de frecuencia, porcentaje válido y acumulativo para factores como: tabaquismo, estadio clínico, hematuria, masa palpable, dolor, calcio sérico, pérdida de peso, grado histológico, y tipo histológico. Cálculo de la media y desviación estándar. Así como la evaluación del riesgo estimado (razón de momios) para estadio patológico, grado histológico, pérdida de peso, masa palpable, dolor, calcio sérico, y tipo histológico con 95\% de intervalo de confianza. Además se realizó análisis de sobrevida de Cutler-Ederer para estadio patológico.

\section{RESULTADOS}

Se analizaron en el presente estudio un total de 96 expedientes clínicos de pacientes atendidos en el servicio de Urología del Hospital de Especialidades CMN SXXI durante 5 años (enero de 1998 a diciembre de 2002). Solamente 52 pacientes cumplieron con los criterios de inclusión, de ellos 28 fueron hombres y 24 mujeres, con una media de edad de 59,85 (35-84) desviación estándar 11,41.

El Carcinoma de células claras se presentó en 94,2\%, carcinoma de células cromófobas en 1,9\%, y otros en $3,8 \%$.

El tabaquismo estuvo presente en 27 pacientes (51,9\%). El tabaquismo no fue significativo estadísticamente en los pacientes que fallecieron ( $p=0,224)$, los tumores mayores de $7 \mathrm{~cm}$ estuvieron presentes en todos los pacientes que fallecieron, la presencia 
de dolor y masa palpable estuvieron presentes en 13 y 11 de los 20 pacientes que murieron (OR: 3,095 y $11,815)$ respectivamente.

El calcio mayor de $10,2 \mathrm{mg} / \mathrm{dl}$ y la pérdida de peso se presentaron en 9 y 14 pacientes asociados a muerte (OR: 2.091 y 16.333) respectivamente con Intervalo de Confianza de 95\%.

En relación al riesgo estimado (OR), las variables más importantes en orden decreciente fueron el estadio patológico, grado histológico, pérdida de peso, masa palpable, dolor, calcio sérico y tipo histológico (Tabla 1).

Tabla 1. Factores pronósticos en orden importancia

\begin{tabular}{lccc}
\hline \multicolumn{4}{c}{ Factores pronósticos } \\
\hline & & \multicolumn{2}{c}{ Intervalo de } \\
confianza 95\% \\
Variable & $\begin{array}{c}\text { Razón de } \\
\text { Momios }\end{array}$ & Bajo & alto \\
\hline I. Estadio patológico & 16.456 & 3.521 & 66.910 \\
II. Grado histológico & 16.379 & 3.670 & 67.341 \\
III. Pérdida de peso & 16.333 & 3.954 & 67.473 \\
IV. Masa palpable & 11.815 & 2.691 & 51.877 \\
V. Dolor & 3.095 & 0.966 & 9.920 \\
VI. Calcio sérico & 2.091 & 0.649 & 6.740 \\
VII. Tipo histológico & 1.871 & 1.415 & 2.471 \\
\hline
\end{tabular}

Todos los pacientes se captaron con enfermedad localizada o localmente avanzada: T1NOMO: 8 pacientes $(15,4 \%)$. T2NOMO: 15 pacientes $(28,8 \%)$. T3aNOM0: 22 pacientes $(42,3 \%)$. T3bNOMO: 7 pacientes (13,5\%) (Fig. 1, Tabla 2).

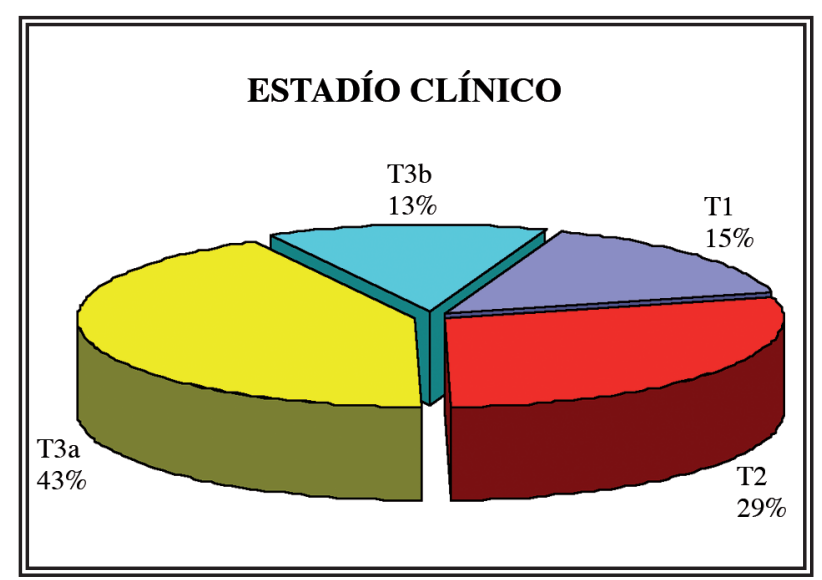

FIGURA 1. Estadios clínicos de los pacientes estudiados.
Tabla 2. Clasificación TNM International Union Against Cancer (UICC), 1997

\section{T: Tumor primario}

Tx. $\quad$ No se puede evaluar el tumor primario.

TO. No existen signos de tumor primario

T1. Tumor de diámetro máximo menor o igual a $7 \mathrm{~cm}$ limitado al riñón.

T2. Tumor de diámetro máximo mayor de $7 \mathrm{~cm}$ limitado al riñón.

T3. Tumor que se extiende a las venas principales o invade la glándula suprarrenal o los tejidos perirrenales, pero sin atravesar la fascia de Gerota.

T3a. Tumor que invade la glándula suprarrenal o los tejidos perirrenales, pero sin atravesar la fascia de Gerota.

T3b. Tumor que se extiende de forma importante en las venas renales o cava por debajo del diafragma.

T3c. Tumor que se extiende de forma importante en la vena cava por encima del diafragma.

T4. Tumor que atraviesa la fascia de Gerota.

\section{N: Ganglios linfáticos regionales}

Nx. No se pueden evaluar los ganglios linfáticos regionales.

NO. No se demuestran metástasis ganglionares regionales.

N1. Metástasis en un único ganglio linfático regional.

N2. Metástasis en más de un ganglio linfático regional.

\section{M: Metástasis a distancia}

Mx. No se puede evaluar las metástasis a distancia.

MO. No hay metástasis a distancia.

M1. Metástasis a distancia.

Fuente: Walsh PC, Kavoussi LR, Novick AC, et al. Renal Tumors. Campbell's Urology 2002; 75: 2699.

La invasión a la vena renal se presentó en 8 pacientes $(15,4 \%)$. La hematuria en 29 pacientes $(55,8 \%)$, masa palpable en 14 pacientes $(26,9 \%)$, dolor en 25 pacientes $(48,1 \%)$ y la triada clásica sólo se presentó en 7 pacientes $(12,7 \%)$. El tamaño del tumor fue mayor de $7 \mathrm{~cm}$. en 43 pacientes $(82,7 \%)$. La frecuencia del grado histológico fue de: $3,8 \%, 32,7 \%, 30,8 \% 32,7 \%$ para los grados: 1,2 , 3 , y 4 de Fuhrman respectivamente (Tablas 3 y 4 , Fig. 2).

Un calcio sérico mayor de 10,2 mg/dl se observó en 9 pacientes (17,3\%), y pérdida de peso de más de $10 \%$ en los últimos 6 meses se presentó en 14 pacientes $(27 \%)$.

Del total de pacientes estudiados durante los 5 años 32 viven sin metástasis después de nefrectomía radical $(61,5 \%)$ y 20 pacientes $(38,5 \%)$ murieron por metástasis, de ellos 16 tuvieron metástasis pulmonar (80\%), 3 con metástasis cerebral (15\%) y 1 
Tabla 3. Clasificación del sistema de Fuhrman para el grado nuclear en cáncer renal

\begin{tabular}{lcll}
\hline Grado & Tamaño del núcleo & Bordes del núcleo & Nucléolo \\
\hline 1 & $10 \mathrm{~mm}$ & Uniforme & Ausente \\
2 & $15 \mathrm{~mm}$ & Irregular & Pequeño \\
3 & $20 \mathrm{~mm}$ & Irregular & Prominente \\
4 & $>20 \mathrm{~mm}$ & Multilobulado & Prominente, corpúsculos de cromatina \\
\hline
\end{tabular}

Fuente: Fuhrman et al. Prognostic significance of morphologic parameters in renal cell carcinoma. Am J Surg Pathol 1982;6:655-663.

Tabla 4. Frecuencia de presentación por grado nuclear

\begin{tabular}{lccc}
\hline \multicolumn{4}{c}{ Grado nuclear de Furhman } \\
\hline $\begin{array}{l}\text { Grado de } \\
\text { Furhman }\end{array}$ & $\begin{array}{c}\text { Número } \\
\text { pacientes }\end{array}$ & $\begin{array}{c}\text { Porcentaje } \\
\text { neto }\end{array}$ & $\begin{array}{c}\text { Porcentaje } \\
\text { acumulado }\end{array}$ \\
\hline Grado I & 2 & 3,8 & 3,8 \\
Grado II & 17 & 32,7 & 36,5 \\
Grado III & 16 & 30,8 & 67,3 \\
Grado IV & 17 & 32,7 & 100,0 \\
Total & 52 & 100,0 & 100,0 \\
\hline
\end{tabular}

paciente con metástasis ósea (5\%). El promedio de sobrevida fue de 18,0 meses a partir del diagnóstico de metástasis.

De acuerdo a la sobrevida de los pacientes con cáncer renal se observó con respecto al estadio patológico una media de sobrevida de 52,45 meses para T1, de 48,31 meses para T2, 11,25 meses para T3a y de 8,25 meses para T3b.

\section{DISCUSIÓN}

El único factor epidemiológico reconocido en la literatura en cáncer renal no urotelial es el tabaquismo, en nuestro estudio no encontramos una diferencia significativa entre los pacientes que tenían tabaquismo intenso y los que no lo tenían. Aunque el tabaquismo está presente en $30 \%$ de los canceres renales según la literatura revisada ${ }^{3}$, en el presente estudio se encontró en el 59,2\% de los casos.

La presencia de hematuria, dolor y masa palpable e incluso la tríada clásica tienen relación con la frecuencia reportada en la literatura ${ }^{5,9,18}$; y dentro de nuestro estudio se puede apreciar que todavía son frecuentes los tumores de gran tamaño a diferencia de lo reportado por Hamdy et al. ${ }^{5}$ en donde se establece que para países desarrollados el diagnóstico de tumores menores de $4 \mathrm{~cm}$ ocurre en el $30 \%$ de los casos y obedece a hallazgos incidentales en estudios de TAC y USG tomados por sintomatología no relacionada ${ }^{8}$.

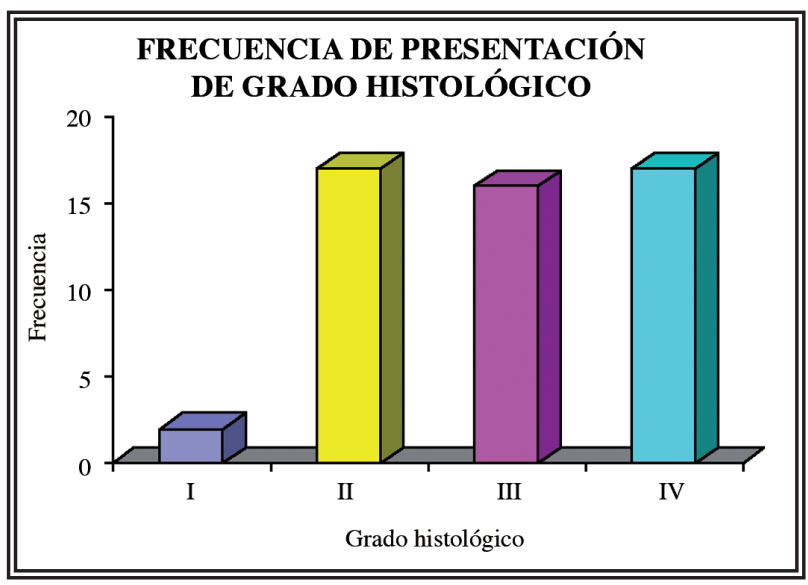

FIGURA 2. Frecuencia de presentación por grado nuclear.

En el presente estudio sólo se incluyeron pacientes libres de enfermedad metastásica al momento del diagnóstico, y se excluyeron por presentarla aproximadamente $46 \%$ de los pacientes inicialmente captados, dato similar al encontrado en la literatura en donde se habla de que $35 \%$ de los pacientes con cáncer renal tendrán metástasis al momento del diagnóstico ${ }^{13,17}$

Gelb et al., reportaron la hipercalcemia como factor importante en la sobrevida de los pacientes con cáncer $\mathrm{renal}^{8}$, en nuestro estudio se obtuvo que 9 de 20 pacientes fallecieron con cifras de calcio sérico mayores de $10,2 \mathrm{mg} / \mathrm{dl}$ por lo que existe una tendencia como factor adverso pero no se logró demostrar estadísticamente.

Los factores de mayor peso pronóstico encontrados en el este estudio son el estadio clínico y el grado nuclear como se reporta en los trabajos realizados por Ozer et al. y Leibovich, sin embargo la pérdida de peso, masa palpable y el dolor demostraron tendencias adversas para el pronóstico de los pacientes, esto tal vez por ser variables relacionadas con el tamaño tumoral y por ende con un estadio clínico avanzado, provocando un impacto en el curso de la enfermedad como factores dependientes. 
La mortalidad de los estadios T3a y T3b es alta y el tiempo hasta la muerte es acelerado a pesar de haberse realizado la nefrectomía radical, lo que orienta a establecer que se subestadifica la enfermedad, y que muy probablemente la posibilidad de micrometástasis en éstos pacientes haya estado presente incluso antes de haber acudido por primera vez a consulta. De aquí la importancia de establecer el diagnóstico de forma más temprana, ya que se observa que en nuestro medio aún los tumores de gran tamaño son frecuentes.

La determinación precisa de factores pronósticos es un paso esencial para la evaluación del paciente con cáncer renal, no solamente para iniciar nuevos tratamientos adyuvantes sino para predecir la evolución de la enfermedad, en el presente estudio se observó una influencia directa del estadio clínico, el grado nuclear, y los síntomas o signos, como la perdida de peso en el desenlace de los pacientes.

Debido a que en este trabajo sólo se tomaron los factores pronósticos como reporte observacional en un grupo de pacientes, es necesario realizar un análisis de la interrelación de factores asociados o combinación de los mismos como pronostico de la enfermedad y establecer la independencia de ellos para que tengan significado estadístico.

El futuro de los factores pronósticos parece estar orientado hacia los marcadores tumorales biológicos que se encuentran en proceso de investigación y a los cuales en nuestro medio no tenemos acceso con facilidad y tal vez sean los factores ideales para determinar el curso y pronóstico en cáncer renal.

\section{CONCLUSIONES}

La utilidad actual de los factores pronósticos es el conocimiento del pronóstico del paciente con cáncer renal. Se han realizado varios estudios con el fin de estandarizar los datos y definir mejor las características pronósticas de la enfermedad. El presente estudio nos permite validar como factores pronósticos en nuestro medio el estadio clínico, grado histológico y tipo histológico.

\section{REFERENCIAS}

1. Landis SH, Murria T, Bolden S, Wingo PA. Cancer statistics. Cancer J Clin. 1999;49(1):8-31.

2. Yu MC, Mack TM, Hanisch R. Cigarette smoking, obesity, diuretic use, and coffee consumption as risk factors for renal cell cancer study. Int J Cancer. 1995;60:194-198.
3. Waters WB, Richie JP. Aggressive surgical approach to renal cell carcinoma: review of 130 cases. J Urol. 1999;122(3):306-309.

4. Hamdy FC, Baster JW, Neal DE, et al. Management of urologic malignancies. J Urol. 2002; 19: 273-275.

5. Fekak H, Bennani S, Taha A, Rabii R, Joual A, Sarf S, et al. Kidney cancer: report of 170 cases. Ann D Urologie. 2001; 35(5):249-256.

6. Figlin RA. Renal cell carcinoma: management of advanced disease. J Urol. 1999;161(2):381-386.

7. Leibovich BC, Zisman A, Figlin RA, et al. Current staging of renal cell carcinoma. Urol Clin North Am. 2003; 30: 1-18.

8. Méjean A, Oudard S, Thiounn N. Prognostic factors of renal cell carcinoma. J Urol. 2003;169:82 1-827.

9. Thrasher JB, Paulson DF. Prognostic factors in renal cancer. Urol Clin North Am. 1993;20(2):247-262.

10. Walsh PC, Kavoussi LR, Novick AC. Renal tumors. J Urol. 2002;75:2672-2721.

11. Baird AD, Woolfenden KA, Desmond AD, Fordham MV, Parsons $\mathrm{KF}$. Outcome and survival with nonsurgical management of renal cell carcinoma. BJU Int. 2003;91(7):600-607.

12. Ozer E, Yörüko€lu K, Sagol O, Mungan U, Demirel D, Tüzel E, et al. Prognostic significance of nuclear morphometry in renal cell carcinoma. BJU Int. 2002;90(1):20-25.

13. Vasselli JR, Yang JC, Linehan WM, White DE, Rosenberg SA, Walther MM. Lack of retroperitoneal limphadenopathy predicts survival of patients with metastatic renal cell carcinoma. J Urol. 2001;166(1):68-72.

14. Gö€üfl C, Baltaci S, Bedük Y, Sahinli S, Küpeli S, Gö€üfl O. Isolated local recurrence of renal cell carcinoma after radical nephrectomy: experience with 10 cases. Urol. 2003;61(5):926929.

15. Patel MI, Simmons R, Kattan MW, Motzer RJ, Reuter VE, Russo P. Long-term follow-up of bilateral sporadic renal tumors. Urol. 2003;61(5):921-927.

16. Vaydya W, Ciancio G, Soloway M. Surgical techniques for treating a renal neoplasm invading the inferior vena cava. J Urol. 2003;169(2):435-444.

17. Zisman A, Pantuck AJ, Chao DH, Wieder JA, Dorey F, Said JW, et al. Renal cell carcinoma with tumor thrombus: Is cytoreductive nephrectomy for advanced disease associated with an increased complication rate. J Urol. 2002;168(3):962-967.

18. Filipas CS, Michaelis J, Roth S, et al. Screening for renal cell carcinoma using ultrasonography: a feasibility study. BJU Int. 2002;91(7):595-599.

19. Palapattu GS, Pantuck AJ, Dorey F, Said JW, Figlin RA, Belldegrun AS. Collecting system invasion in renal cell carcinoma impact and prognosis and future staging strategies. J Urol. 2003;170(3):768-772

20. Gill IS, Matin SF, Desai MM, Kaouk JH, Steinberg A, Mascha $\mathrm{E}$, et al. Comparative analysis of laparoscopic versus open partial nephrectomy for renal tumors in 200 patients. J Urol. 2003:170(1):64-68.

Correspondencia autor: Dr. V.H. Rodríguez-Jasso Servicio de Urología. Hospital de Oncología Centro Médico Nacional Siglo XXI. Instituto Mexicano del Seguro Social. Avda. Cuauhtemoc, n ${ }^{\circ} 330$ Col. Doctores - 06720 Cuauhtemoc D.F. Méjico. Tel.: +50356276900

E-mail autor: uromail@gmail.com Información artículo: Original - Cáncer renal Trabajo recibido: mayo 2007

Trabajo aceptado: julio 2007 